Erschienen in: Brünner, Gisela/Fiehler, Reinhard/Kindt, Walter (Hrsg.): Angewandte Diskursforschung. Band 1: Grundlagen und Beispielanalysen. - Opladen: Westdeutscher Verlag, 1999. S. 215-232.

\title{
Selbstdarstellung im öffentlichen und beruflichen Gespräch
}

\author{
Carmen Spiegel/Thomas Spranz-Fogasy
}

\section{Zusammenfassung}

In diesem Aufsatz möchten wir das Phänomen der Selbstdarstellung in verbaler Interaktion behandeln und die Relevanz verdeutlichen, die diesem Phãnomen insbesondere in institutionellen und öffentlichen sozialen Situationen zukommt. Wir stellen zunächst unseren Begriff der 'Selbstdarstellung' vor, der eng im Zusammenhang mit unserem Konzept der verbalen Interaktion steht, das wir kurz erläutern werden (Abschnitte 1 und 2). Danach gehen wir auf die Rolle der Selbstdarstellung in öffentlichen und institutionellen Gesprächen ein (3). Bevor wir verschiedene Arten der Selbstdarstellung an Gesprächsbeispielen präsentieren, um zu zeigen, wie Selbstdarstellung interaktiv gestaltet und wirksam wird, wollen wir auf das Pendant der Selbstdarstellung, die Fremddarstellung, hinweisen (4-6). Wir möchten bereits hier betonen, daß wir für das Phänomen der Selbstdarstellung nur sensibilisieren können. Patentrezepte für Selbstdarstellungen in den verschiedenen Lebenssituationen gibt es aufgrund der Unterschiede in den Persönlichkeiten und Situationen nicht, zu sehr hängt die Selbstdarstellung und ihre Wirkungsweise von den jeweiligen Kontexten und Umständen ab; darauf gehen wir am Ende unseres Aufsatzes ein (7).

\section{Zum Konzept der Selbstdarstellung}

Unter Selbstdarstellung verstehen wir all diejenigen Aspekte sprachlichen und nichtsprachlichen Handelns, mit denen Menschen im Gespräch einander ihre kulturellen, sozialen, geschlechtlichen und individuellen Persönlichkeitseigenschaften präsentieren. In den meisten Äußerungen von Gesprächsteilnehmerlnnen finden sich - beabsichtigt oder unbeabsichtigt - vielfältige Spuren ihrer jeweiligen Persönlichkeiten, die mit den unterschiedlichen Formen der Selbstdarstellung ausgedrückt werden. In der Alltagssprache gibt es eine Fülle von Charakterisierungen und Vergleichen zur Beschreibung solcher Selbstdarstellungen in bezug auf Sprechstile (kindlich, altväterlich, dozierend, höflich, arrogant, blumig), in bezug auf verschiedene soziale (Rollen-)Stile (die spricht wie eine Lehrerin, wie eine Marktfrau, wie eine Mutter) und zur Beschreibung der verschiedenen Arten, Gesprächsaufgaben anzugehen und zu bewältigen (bedächtig, forsch, zögerlich, rücksichtslos, umsichtig, sich drücken etc.). Selbstdarstellungen können dabei bewußt präsentiert oder regelrecht in Szene gesetzt werden, meist sind sie jedoch ein weniger bewußter Bestandteil von Interaktion. Sandig (1986) unterscheidet für stilistische Selbstdarstellung einen bewußt kontrollierbaren und deshalb intentionalen Ausdruck der Stilisierung vom nicht kontrollierbaren und deshalb symptomatischen Ausdruck. Diese Unterscheidung gilt auch für Selbstdarstellung allgemein.

Selbstdarstellung steht, obwohl durchgehende Interaktionsaufgabe, meist nicht im Zentrum der Aufmerksamkeit. In bestimmten Situationen oder in bestimmten Phasen von Gesprächen kommt der Selbstdarstellung aber auch eine besondere Bedeutung zu: in Bewerbungsgesprächen, wenn es darum geht, die eigenen Fähigkeiten 'ins rechte Licht' zu rücken, in Prüfungsgesprächen, wenn es darum geht, sich kompetent zu zeigen, oder in Verhandlungen, wenn eine Vertrauensbasis hergestellt oder Durchsetzungsfähigkeit demonstriert werden muß. In solchen Situationen greifen GesprächsteilnehmerInnen vielfach auf bestimmte kommunikative Muster zurück, auf spezifische Arten des Sprechens, die erfolgversprechend scheinen: zurückhaltendes, vermittelndes oder aggressives Sprechen und Interagieren, bestimmte Sprachregister und feste Formulierungen, besondere Intonationen und Akzentuierungen; auch Nonverbales wie Gestik, Mimik, Blickkontakt 
oder Körperhaltung sind Bestandteil selbstdarstellender kommunikativer Muster, ja sogar die Kleidung - man denke an die Aufmachung von Punks, an Büro- und Freizeitkleidung, an bevorzugte Bekleidungsmarken etc. - ist ein Element der Selbstdarstellung. ${ }^{1}$

Selbstdarstellung zeigt sich zum einen in den Inhalten, durch das Was des Handelns, also durch das, was explizit gesagt und was damit für die jeweils im Mittelpunkt stehende Handlungsaufgabe des Gesprächs getan wird; so beispielsweise, was ich als Problem ansehe und darstelle, was mir bei einem Bewerbungsgespräch wichtig erscheint und was ich lieber weglasse, worauf ich bei einer Verhandlung Wert lege und was ich als unwichtig abtue. Selbstdarstellung umfaßt aber vor allem auch die besondere Art der Durchführung von Äußerungen, das Wie dieses Handelns $^{2}$ : welchen Gesprächsstil oder welches Sprachregister ich wähle, welche Strategie ich anwende etc. Die Unterscheidung zwischen dem Was und dem Wie einer Handlung ist jedoch zumeist eine analytische und im realen Handeln oft nicht einfach zu erkennen.

\section{Die gesprächsanalytische Konzeption verbaler Interaktion}

Selbstdarstellung bezieht sich auf verschiedene Dimensionen und Realisierungsebenen verbaler Interaktion. Gespräche kommen durch die Aktivitäten der Gesprächsbeteiligten zustande und werden gemeinsam durchgeführt. Zu allen Zeitpunkten und in allen Hinsichten eines Gesprächs sind es die Gesprächsteilnehmerlnnen, deren sprachliche, parasprachliche und nonverbale Aktivitäten das Gespräch organisieren, seine Inhalte bestimmen, die jeweils besonderen Handlungsaufgaben aus- und bearbeiten oder die Gesprächsrollen und deren Beziehungen zueinander gestalten.

Kallmeyer und Schütze haben analytisch sechs, miteinander wechselwirkende Ebenen der verbalen Interaktion bestimmt, auf denen GesprächsteilnehmerInnen solche Konstitutionsleistungen erbringen: die Ebene der Gesprächsorganisation, der Handlungskonstitution, der Sachverhaltsdarstellung, der sozialen Identitäten und Beziehungen, der Interaktionsmodalitäten sowie die Ebene der Reziprozitätsherstellung. ${ }^{3}$ Auf allen diesen Ebenen realisieren die Beteiligten Muster des Handelns, die jedoch individuell variiert werden können und so zur Selbstdarstellung beitragen. Beispielsweise gelten auf der Ebene der Gesprächsorganisation unterschiedliche Anforderungen, je nachdem, ob es sich um Beratungs- oder Therapiegesprä-

' In der Linguistik wurden Aspekte der Selbstdarstellung im Rahmen der Stilistik- bzw. Stilisierungsforschung (Sandig 1986, Hinnenkamp/Selting 1989, Sandig/Selting 1997), im Zusammenhang mit Beziehungsaspekten (Holly 1979,1990) sowie in Verbindung mit der Gestaltung sozialer Beziehungen (Schmitt 1992, Schwitalla 1996) bzw. im Zusammenhang mit sozialen Stilen (Kallmeyer 1995, Keim 1995) beschrieben.

2 Ältere Konzepte, wie z.B. das linguistisch-pragmatische Stilkonzept, charakterisieren die Typik des Handelns weitgehend als unabhängig von ihrer Modalisierung (z.B. Sandig 1986); demgegenüber sieht eine ethnographisch orientierte Stilforschung im Sinne Gumperz' (1982), daB mit "Unterschieden der Handlungsrealisierung [ = Modalisierung] auch unterschiedliche Bedeutungen und Funktionen verbunden sind" (Kallmeyer 1995, 6), beide Aspekte also wechselwirken.

3 Vgl. dazu Kallmeyer/Schütze 1976 und Kallmeyer 1988, eine zusammenfassende Darstellung findet sich auch in Spranz-Fogasy (1997), Abschnitt I.3. 
che, Verhandlungen, Lehrgespräche, Diskussionen oder Verkaufsgespräche handelt: Einmal verwenden die Gesprächsbeteiligten eher komplexe Äußerungen, um sich z.B. als eloquent oder kompetent zu präsentieren; ein anderes Mal sind schnelle Sprecherwechsel typisch, wenn es z.B. darauf ankommt, zu demonstrieren, daß man besonders schlagfertig ist oder rasch reagieren kann; in einer Gesprächssituation halten die SprecherInnen Unterbrechungen und Korrekturen für opportun, um zu demonstrieren, daß sie ganz bei der Sache, Vorgesetzte oder kompetent sind, in einer anderen Situation sind Rederechte sakrosankt, und man gilt als unhöflich oder gar unverschämt, wenn man trotzdem unterbricht.

Oder bei der Realisierung von Handlungsmustern auf der Ebene der Handlungskonstitution orientieren sich die Gesprächsteilnehmerlnnen zwar einerseits an Normalvorstellungen über Aktivitätsbestandteile, deren Reihenfolge und damit verbundene Beteiligungsaufgaben, sie zeigen sich jedoch andererseits hinsichtlich Variationen, Expansionen oder Reduktionen einzelner Aspekte relativ flexibel bis hin zu vollständiger Unterlassung von Handlungsaufgaben (vgl. dazu Nothdurft/SpranzFogasy 1991). Systematische Musterkonkordanz ist dabei auf allen Ebenen ebenso relevant für Selbstdarstellung - jemand verhält sich, wie es erwartet wird, sei es bei einer Prüfung oder bei einem Verhandlungsgespräch - wie systematische Verstöße gegen (idealtypische) Mustervorstellungen - jemand präsentiert eine unübliche Verhaltensweise z.B. bei einem Vorstellungsgespräch, um sich von Konkurrenten abzusetzen oder Individualität zu vermitteln; das kann gut ankommen, aber auch abgelehnt werden und damit dem Handelnden zum Nachteil gereichen.

\section{3. Öffentliche und berufliche Gesprächssituationen}

Öffentliche und berufliche Gespräche, wie z.B. Interviews, Diskussionen, Vorträge, Verkaufs- und Verhandlungsgespräche, Beratung oder Schlichtung, Lehr- oder Instruktionsgespräche, Prüfungs-', Bewerbungsgespräche oder Besprechungen, ${ }^{4}$ sind aufgabenbezogen und erfolgsorientiert. Es geht in ihnen darum, Ansichten vorzutragen oder Angebote zu machen, sie zu prüfen, anzunehmen oder ggf. zu verwerfen; es geht darum, Entscheidungen vorzubereiten und zu treffen, Probleme zu verdeutlichen, Konflikte zu beseitigen, Lösungen zu entwickeln, Kenntnisse zu vermitteln (oder zu prüfen) usw. Dabei müssen die Beteiligten Kompetenz demonstrieren, sich als glaubwürdig und/oder als vertrauenswürdig darstellen.

Selbstdarstellung ist in diesen Gesprächen immer auf solche Interaktionsaufgaben hin zu gestalten, und ihr 'Erfolg' ist immer daran zu bemessen. Das gilt auch für den Fall destruktiven oder obstruktiven Handelns, da ja auch damit - wenn auch nicht kooperativ - Aufgaben bearbeitet und Ziele verfolgt oder erreicht werden können. Je nach Handlungsaufgabe und Zielvorstellung können dementsprechend ganz unterschiedliche und teils gegensätzliche Elemente der Selbstdarstellung funktional sein. In vielen Gesprächssituationen zeigt sich darüber hinaus, daß die zur Bewältigung der Handlungsaufgaben erforderlichen Eigenschaften einander widersprechen können. So sind z.B. in Gesprächen mit Verhandlungscharakter

4 Es gibt bislang u.W. keine umfassende Typologie öffentlicher oder beruflicher Gespräche, die Liste ist daher zufällig. Hinweise für eine solche Typologie finden sich in Henne/Rehbock 1982, Hundsnurscher 1994, Munsberg 1994 sowie in neueren Publikationen zur Fachsprachen- und Textlinguistik. 
sowohl Durchsetzungsvermögen als auch Entgegenkommen erforderlich und müssen entsprechend plaziert werden. Fingerspitzengefühl für die Gesprächssituation, aber auch für die Gesprächspartnerlnnen ist gefragt.

Mit der Aufgabenbezogenheit öffentlicher und beruflicher Gespräche ist zugleich eine symmetrische oder komplementäre Beteiligungsweise des oder der einzelnen verbunden. GesprächsteilnehmerInnen können ihre jeweiligen Aufgaben erfüllen, problematisieren oder nicht erfüllen bzw. gar verweigern. Sie können dies jeweils auf schnellstem Wege tun, sie können dabei aber auch umständlich agieren oder unentschieden. So ist es beispielsweise in beratenden oder therapeutischen $\mathrm{Ge}$ sprächen die Aufgabe des Ratgebenden/Therapeuten, sich zunächst mit dem Klienten über dessen Probleme bzw. Beschwerden zu verständigen, um zu einer Problemdefinition bzw. Diagnose zu gelangen. Häufige Unterbrechungen oder direktives Frageverhalten können sich in diesem Zusammenhang schnell zum Bild eines ungeduldigen, fall- statt personenorientierten ${ }^{5}$ Beraters/Therapeuten fügen. Neben solchen gesprächsorganisatorischen oder Sprechhandlungs-Ressourcen sind auch auf anderen Ebenen der Interaktionsorganisation viele weitere strukture/le Möglichkeiten für Selbstdarstellung vorhanden, die mit verbalen, para- und nonverbalen Mitteln genutzt werden. Auf der Modalitätsebene beispielsweise signalisieren die Gesprächsbeteiligten Ernsthaftigkeit oder Entspanntheit, oder sie äußern auf der Beziehungsebene z.B. Sympathiebekundungen oder Verärgerung über die Gesprächspartnerln.

Selbstdarstellungsmittel und -strategien sind dabei nicht zwangsläufig stabil, sie variieren und wechseln je nach Stand der Interaktion und den spezifischen Interessen der einzelnen, je nach den Reaktionen der anderen Beteiligten und dem weiteren Gesprächsverlauf. Gesprächsbeteiligte können ihre Selbstdarstellung so den jeweiligen Umständen anpassen.

Eine ganze Reihe von Selbstdarstellungsmitteln und -strategien sind auch stark an den Ablauf von Interaktionen geknüpft und an bestimmte Positionen bzw. Gesprächsrollen gebunden. Für die Selbstdarstellung ist in allen Gesprächen, neben bestimmten Schaltstellen der verbalen Interaktion, die Eröffnungsphase besonders relevant, werden doch hier Situations- bzw. Interaktionstypik hergestellt, Beteiligungsrollen und ihre Beziehungen ausgehandelt, geregelt und oft weit vorausgreifend festgelegt. Umgangssprachlich spricht man vom ersten Eindruck, oder man sagt, daß es von Anfang an schief lief.

Im Verlauf von Gesprächen finden sich noch weitere Schaltstellen, an denen inhaltliche und handlungsspezifische Bündelungen erfolgen und die Beteiligungsqualitäten sich ändern. In aufgabenbezogenen Interaktionstypen, wie es viele institutionelle und öffentliche Gespräche sind, haben solche Schaltstellen oft ihren festen Platz: Darstellungen müssen zusammengefaßt, Probleme und Konflikte definiert werden. Im Zusammenhang solcher Reduktionen ${ }^{6}$ ändern sich dann vielfach auch die Beteiligungsqualitäten der Gesprächspartner - mit all den Möglichkeiten und Risiken, die sich mit einer neuen 'Rolle' oder einer neuen Präsentation des Selbst ergeben: In Beratungsgesprächen z.B. wechselt der Berater vom auf-

5 Vgl. zu dieser Unterscheidung die Charakterisierungen in Rogers 1988 und Balint 1965; vgl. auch Spranz-Fogasy 1992.

6 Zur Notwendigkeit und Funktion von Reduktionen in aufgabenbezogenen Interaktionen vgl. Deppermann/Spranz-Fogasy (1997). 
merksamen Zuhörer zum Problemdefinierenden und weiter zum aktiv Ratschlagenden mit der Gefahr, sein Gegenüber mit einem dozierenden Ton einzuschüchtern, oder in Schlichtungsgesprächen wendet sich der zunächst eher rezeptive Schlichter der aktiven Lösungssuche zu mit dem Risiko der Parteilichkeit; komplementär verhalten sich dann die jeweils anderen Beteiligten. Besonders augenfällig ist das Beispiel ärztlich-therapeutischer Kommunikation: Hatte ein Arzt sich bei der vom Patienten vorgetragenen Beschwerdenschilderung zunächst abwartend verhalten, so können eine terminologisch überfrachtete Diagnosestellung bzw. -mitteilung und ein direktives Verordnungshandeln diesen Eindruck des Patienten vom verständnissuchenden Arzt verkehren?

\section{Die Verwobenheit von Selbstdarstellung und Fremddarstellung}

In unserem Beitrag beschränken wir uns im wesentlichen auf die Beschreibung von Selbstdarstellungsphänomenen. Dennoch möchten wir hier kurz auf die Verflechtung von Selbst- und Fremddarstellung eingehen, denn die Gesprächsteilnehmerinnen präsentieren sich selbst in interaktiver Weise und kontextspezifisch, d.h. im Hinblick auf ihre jeweiligen Partnerinnen und in Reaktion auf sie.

Das kann in verschiedenen Stufungen geschehen, was wir an Sprecher A und Sprecherin B erläutern wollen:

(1) Sprecher A stellt nicht nur sich selbst dar, sondern macht neben seiner Selbstdarstellung - oder durch diese - deutlich, als was er die jeweils anderen GesprächsteilnehmerInnen sieht: Er präsentiert komplementär oder kontrastiv zu seiner Selbstdarstellung eine Fremddarstellung von Sprecherin B.

(2) Im weiteren Gesprächsverlauf rezipiert Sprecherin B einerseits die Selbstdarstellung von $A$; sie modifiziert ihr Fremdbild, das sie von $A$ hat, entsprechend seiner Selbstdarstellung. Darüber hinaus nimmt sie aber auch wahr, wie Sprecher A in seiner Fremddarstellung sie selbst darstellt.

(3) Sprecherin B wiederum kann auf das Bild reagieren, das A von ihr zeichnet, indem sie eine entsprechende Selbstdarstellung präsentiert, und sie kann auf die zuvor präsentierte Selbstdarstellung von A reagieren, indem sie eine entsprechende Fremddarstellung vermittelt.

Häufig werden Selbstdarstellung und Fremddarstellung eng miteinander verwoben präsentiert wie im nachfolgenden Beispiel (vgl. Spiegel 1995). In dem kleinen Ausschnitt aus einem Streit zwischen zwei Partnern zeigt sich diese Verwobenheit; es ist hier Sprecher A, der auf eine vorangegangene Selbst- und Fremddarstellung von Sprecherin B reagiert (eine Erklärung der Transkriptionszeichen findet sich am Ende dieses Beitrags):

A: du behandelst mich immer gleich verstehst/ du du hast halt so=n festes bild * ich meine halt wirklich daß ich noch

7 Vgl. Spranz-Fogasy 1988 und 1992. 
halbwegs flexibel bin und kann auch mal dir (gegenüber) auf was reagieren und bei dir mein ich des halt nich

In seinem ersten Äußerungsteil (du behande/st mich immer gleich) spricht A die Behandlungsweise von Sprecherin $B$ an, die, nach Meinung von $A$, auf einem bestimmten Fremdbild der Sprecherin B über den Sprecher A beruht (du du hast halt $s o=n$ festes bild). Das heißt, Sprecher A reklamiert die Art der Behandlung durch Sprecherin B, er vermutet, daß diese Art der Behandlung auf einem bestimmten Fremdbild der Sprecherin B über ihn beruht. Er reklamiert aber nicht nur das Fremdbild von $B$, sondern er versucht es auch zu bearbeiten, indem er etwas über sich selbst sagt: er präsentiert eine explizite Selbstdarstellung (ich meine halt wirklich daß ich noch halbwegs flexibel bin und kann auch mal dir (gegenüber) auf was reagieren).

Darüber hinaus präsentiert Sprecher A aber auch eine Fremddarstellung seiner Gesprächspartnerin; einmal, indem er zu Beginn seiner Äußerung etwas über ihr Verhalten ihm gegenüber sagt (du behande/st mich immer gleich), und zum anderen, als er am Ende seiner Äußerung eine Einschätzung über ihr Verhalten formuliert (und bei dir mein ich des halt nich) kontrastiv zu seiner Selbsteinschätzung. Anders formuliert: Sprecher A präsentiert eine explizite Fremddarstellung seiner Gesprächspartnerin B komplementär zu seiner eigenen Selbstdarstellung, diese wiederum ist eine Kontrastdarstellung zu der Fremddarstellung, die er bei Sprecherin $B$ über seine Person vermutet. Hier zeigt sich deutlich die Verwobenheit von Selbstund Fremddarstellung. ${ }^{8}$

So deutlich und explizit finden sich Selbst- und Fremddarstellungen und deren Bearbeitungen jedoch nur selten in institutionellen und öffentlichen Gesprächen, weder der situative Rahmen noch die Regeln der Höflichkeit lassen dies i.d.R. zu.

\section{Die Arten der Selbstdarstellung}

Selbstdarstellungen kommen in den unterschiedlichsten Formen vor, idealtypisch zeigen sie sich auf drei Weisen:

- als explizite Selbstaussagen, so z.B. in Formulierungen wie ich als realist, pazifistin, privatmensch loder: ich meine halt wirklich daß ich noch halbwegs flexibel bin) etc.;

- als Eigenschaften sprachlicher und sprachbegleitender Aktivitäten (daß jemand lautstark lamentiert, lobt oder bittet, statt zu fordern; sich in Erzählungen auf bestimmte Weise präsentiert etc.) und schließlich

- Vergleichbar unterscheidet Fiehler $(1990,30)$ analytisch drei systematisch aufeinander bezogene Teilaufgaben in der "Prozessierung einer sozialen Beziehung": Selbstpräsentation, Bewertung der Selbstpräsentation der anderen Person und Bewertung der Stellungnahme der anderen Person zur eigenen Selbstpräsentation. 
als übergreifende Eigenschaften des Gesprächshandelns (daß sich z.B. jemand besonders freundlich/unfreundlich über ein ganzes Gespräch hinweg gibt oder eine bestimmte Interaktionsrolle einnimmt ${ }^{9}$ ).

Im folgenden zeigen wir anhand von Beispielen aus einer umweltpolitischen Diskussion über umweltschädigendes versus umweltschonendes Verhalten zunächst lokale Arten der Selbstdarstellung zweier Diskussionsteilnehmer (5.1). Das zweite Beispiel aus einer Schlichtungsverhandlung zeigt dann - in bezug auf eine einzelne Person - übergreifende Eigenschaften des gesprächsweisen Handelns (5.2), die im Vergleich zu den lokalen Selbstdarstellungsmitteln eher global und auf Aspekte der interaktiven Entwicklung und Phasierung hin charakterisiert werden müssen.

\subsection{Beispie/ 1: Explizite Selbstaussagen und Eigenschaften sprachlicher Aktivitäten - Gegenspieler in der umweltpolitischen Diskussion}

Selbstdarstellung hat in Small Talk oder in einer anderen informellen Situation einen anderen Stellenwert als in umweltpolitischen Diskussionen. In deren Kontext kommt es darauf an, einerseits eine 'politische' Position in der Umweltdiskussion darzulegen, andererseits den einer solchen Debatte inhärenten Normen und Erwartungen gerecht zu werden - und dabei nicht an Glaubwürdigkeit zu verlieren.

A: äh für mich realistisch erkennen hei"Bt zum beispiel- * daß* sie sprachen vorhin von der entfernung * muB jemand in münchen leben wie ich beispielsweise und in losing arbeiten * ich fahre jeden tach hundertvierundsechzig kilometer ăh entschuldigung hundertvierundzwanzig kilometer hin und zurück- * mi=m auto- * beeintrăchtige damit- * ãhm die umwelt * a"ber- * für mich ist es halt- * ein stück lebensqualităt in münchen in der sta"dt zu wohnen weil ich eben ein stådtisch geprägter mensch bin * und ich gebe ganz offen zu ich hoffe daB $i c h$ da niemandem hier in dem raume- * zu nahe trete* aber- * ich könnte es für mich- * halt nur sehr schwer vorstellen im lăndlichen raum zu leben * aber da muB i"ch mit fertig werden * bei ihnen wăr=s ganz genauso umgekehrt ich kenn jemanden in losing der pendelt jeden tach nach münchen * der möchte * um=s verrecken nich in münchen

In diesem Gesprächsausschnitt sind verschiedene sprachliche Mittel für die Selbstdarstellung des Sprechers relevant:

- Die Inhalte, die er formuliert: Er redet explizit über sich und sein Tun, bzw. über sein Umweltverhalten (ich fahre jeden tach ... beeinträchtige damit), über seine Ansichten (für mich realistisch erkennen heißt .... weil ich eben ein städtisch geprägter mensch bin). Der Sprecher äußert sich explizit zu seiner Person, und er gibt sich mit dem, was er sagt, zu erkennen: Der Sprecher schildert Aktivitäten, die bestimmte soziale Kategorien aufrufen; ein Pendler, der täglich über hundert Kilometer mit dem Auto zur Arbeit fährt, verhält sich beispielsweise

Vgl. Spranz-Fogasy (1997), Schwitalla (1996). 
nicht gerade umweltschonend. Zugleich stellt er sich auf der Ebene kommunikativen Handelns als offen dar.

- Die Auswahl seiner Inhalte: Er redet über seine Person und schildert aus seiner Perspektive einen Ausschnitt aus seiner Lebens- und Erfahrungswelt. Zur Darstellung der eigenen Position hätte er auch eine andere Auswahl treffen können, wie der Gesprächsausschnitt seiner Kontrahentin zeigt, auf den wir gleich eingehen werden.

- Sein Gesprächsstil: Er schildert seine Aktivitäten in einem erzählenden, teils bekennenden Stil, durchsetzt mit alltagssprachlichen Floskeln wie z.B. etwas ist ein stück lebensqualität, ein städtisch geprägter mensch sein, niemandem zu nahe treten, da muß ich mit fertig werden, etwas ums verrecken nich wollen oder etwas ganz offen zuzugeben. Dem Sprecher geht es darum, sich als 'realistischen und offenen Menschen' zu präsentieren: Durch die szenische Darstellung von Gegenständen aus alltagsweltlichen Zusammenhängen in einem eher nüchternen Stil 'zeigt' der Sprecher, daß er Realist ist, daß er - wie er in einer anderen Äußerung sagt - die 'Welt sieht und erkennt, wie sie ist'.

Daß Sprecher A mehreren verschiedenen Anforderungen gerecht zu werden versucht, zeigt sich an seinen Gesprächsaktivitäten: Auf der Ebene der kommunikativen Beziehungen stellt er sich dar als Mensch, der seine Ansichten aufdeckt (ich gebe ganz offen zul. Auf der inhaltlichen Ebene sucht er sich dann einerseits als umweltbewußt Handelnder zu präsentieren, der andererseits aber auch seinen Neigungen gerecht werden möchte. Er stellt sich als Stadtmensch dar, der es auf dem Land nicht aushalten würde, er rechtfertigt die sich daraus ergebenden (umweltschädlichen) Konsequenzen einerseits durch Signalisieren von Verständnis der Gegenposition (und dadurch implizites Werben um Verständnis für die eigene Position), andererseits dadurch, daß er durch die Art der Darstellung demonstriert, daß er um sein umweltschädigendes Verhalten weiß, und er signalisiert ein schlechtes Gewissen: mit dem Verzögerungselement ähm in seinem Eingeständnis beeinträchtige damit- * ähm die umwelt, mit seiner direkten Rechtfertigung: für mich ist es halt- "ein stück lebensqualität und mit seiner indirekten Rechtfertigung mit Hilfe eines Gegenbeispiels: ich kenn jemand in losing der pendelt jeden tach.

Kurz darauf berichtet Sprecher A noch von Aktivitäten, welche die soziale Kategorie des Umweltbewußten aufrufen - und stellt sich damit gleichzeitig als umweltbewußt dar:

\section{A: ich würde ja gerne umsteigen * auf offentliche verkehrsmittel * innerhalb von münchen fahr ich nu"r mit der ubahn und der esbahn oder mit=m fahrrad *}

Doch für seine Kontrahentin ist sein Verhalten nicht akzeptabel, seine Präsentation als Realist nicht glaubwürdig genug. Durch die Art, auf ihren Kontrahenten zu reagieren, vermittelt sie gerade im Kontrast zu ihrem Gegner eine bestimmte Form der Selbstdarstellung - und der Fremddarstellung:

B: die welt wird zu klein und wir fressen sie buchstablich aus äh und löffeln sie auf und ăh aus und leben vom vom einge- 
machten * das is das problem und in dem kontext muß ich über neue- ãh umgehensweisen des verantwortungs bewußten menschen* im ganzen * ăh * diskutieren und kann nicht kucken wo ich hie" $r=n$ biBchen repariere und vielleicht also- * $n=b i B c h e n$ billiger von münchen nach losing fahre das ist mir vie"l zu kleinkariert und ăh * natü"rlich muß ich ăh- * ne utopie die ich entwerfe is zunăchst mal einfach sowas wie=ne visio:n * und der muß ich mich năhern aber oh"ne ne vision i"mmer nur mit dem * ich sach jetzt mal korrigieren * und rumbastelndazu ham wer eigentlich keine zeit mehr * es is- wirklich nicht radikal genuch *

Im Vergleich zur vorangegangen Äußerung von Sprecher A wird die so ganz andere Art der Darstellung deutlich: Inhalte, Auswahl und Stil sind kontrastiv zum Vorgänger ausgewählt. Sie zeigen eine andere Selbstdarstellung der Sprecherin, die im Kontrast zum negativen Fremdbild steht, das sie über ihren Kontrahenten zeichnet.

- Die Inhalte: Sprecherin B kritisiert den Zustand der Welt, und sie kritisiert ihr Gegenüber indirekt, indem sie seine Handlungsweise negativ bewertet und antithetisch zu ihrem eigenen 'Entwurf' darstellt.

Die Auswahl der verbalisierten Inhalte: Anstelle der Erlebnisse aus der eigenen Erfahrungswelt schildert Sprecherin B metaphorisch - und recht abstrakt - den Zustand der Welt. Ihr Sujet ist ein anderes, es ist g/obaler, wie sie an anderer Stelle sagt.

- Der Stil: B formuliert ihren Gegenstand abstrakter, verwendet viele Metaphern und wird gelegentlich geradezu 'metaphysisch'. Ihre Selbstdarstellung präsentiert sie also oft nicht durch explizite Aussagen, sondern vor allem über diese Eigenschaften ihrer sprachlichen Aktivitäten bzw. allgemein über diesen $\mathrm{Ge}$ sprächsstil.

Beide Sprecher vermitteln in diesen Ausschnitten ihre Sicht von Welt mit unterschiedlichen Mitteln und stellen sich damit unterschiedlich selbst dar. Sie präsentieren zwei verschiedene Typen, den Realisten, der Ausschnitte seiner Lebenswelt vorstellt, und die Utopistin, die mit ihren Visionen die Welt zu retten versucht.

\subsection{Beispiel 2: Übergreifende Eigenschaften des Gesprächshandelns - Der Schlichter zwischen Justiz und Lebensalltag}

Im folgenden Beispiel aus einem Schlichtungsgespräch vor einer baden-württembergischen Vergleichsbehörde ${ }^{10}$ verhandelt der Schlichter Kastner (C) ${ }^{11}$ mit den

10 Zum Interaktionstyp Schlichtung vgl. Spranz-Fogasy (1997), Kap.II.3; zur Institution der badenwürttembergischen Vergleichsbehörde vgl. Spranz-Fogasy (1997), Kap.III.2 sowie allgemein Huttner 1981.

1 Für die Ausschnitte aus Schlichtungsgesprächen werden für die Beteiligten die Siglen $A=$ Klägerpartei, $B=$ Beklagtenpartei sowie $C=$ Schlichter verwendet, die angegebenen Namen sind geändert. 
Konfliktparteien über leichte Körperverletzung, Beleidigung und Drohung. Die Beklagten, Herr und Frau Neumeier (B1 und B2), bestreiten die Vorwürfe nur teilweise, verweisen aber auf Vorfälle, bei denen der Antragssteller, Herr Beck (A), sich Dinge zuschulden kommen ließ. ${ }^{12}$ Die konfliktäre Konstellation wird vom Schlichter in wichtigen Passagen vor allem mit dem Einsatz zweier gegensätzlicher Formen der Selbstdarstellung bearbeitet und schließlich in einen Vergleichserfolg umgemünzt: Zum einen zieht er sich auf seine Rolle als Schlichter zurück und schafft durch die Präsentation formal-juristischer Beurteilungskompetenz Distanz; Kennzeichen hierfür ist ein entsprechendes Sprachregister mit juristischen Floskeln und Fachvokabular und die Verwendung der Hochsprache. Zum anderen paßt sich der Schlichter in bestimmten Gesprächssituationen mit der Verwendung einer dialektalen Variante und eines bestimmten Sprachregisters (Lebensweisheiten) an die umgangssprachliche Lebenswelt der Konfliktparteien an und hebt so die institutionell und rollenspezifisch vorgegebene Distanz auf: Er spricht als Mensch zu seinesgleichen.

Schon zu Beginn der Verhandlung, in seiner ausführlichen Darstellung der Funktion der Vergleichsbehörde und des Klagevorwurfs, werden vom Schlichter beide Formen der Selbstdarstellung eingesetzt, die formelle hier insbesondere, um die institutionelle Qualität des Geschehens zu verbürgen:

C: [...] zur bedeu"tung der vergleichsbehörde für die beiden parteien kurz folgendes wir sind also kein gerischt * * wir kőnnen * keine beweisaufnahme durchführen mer kőnne auch niemanden * also keine zeugen hören oder so irgendetwas * u"nsere aufgabe die wir vom gesetzgeber zu"gewiesen erhalten ist also die [...] einen außergerischtlischen * vergleisch * $a ̈: h$ zu bewerkschtelligen * denn der gesetzgeber möchte also ni"scht * daß bei solchn fällen * hier * groß * der * ka"di angerufen wird [...]

Die Möglichkeit, mit den Konfliktparteien auch im Kontext der Schlichtung in deren lebensweltlicher Umgangssprache zu interagieren, ergibt sich dabei für ihn bereits zu Beginn der Schlichtung aus den - schriftlich vorliegenden - Klagevorwürfen, die er dialektal gekonnt wiedergibt, so u.a.:

$C:[\ldots]$ da"B * der herr neumeier ihn am handgelenk * * angefaßt hătte * und ihm gesacht freundschn allein schon für da"s * könnt isch dir in die fresse schlagn [...]

Im gesamten Verlauf der Verhandlung changiert der Schlichter dann an allen handlungsschematisch oder beziehungsdynamisch wichtigen Stellen zwischen den beiden Präsentationsformen Dialekt und Standard:

- Formal-juristisch bei Nachfragen zum Verfahren:

C: offizialdelikte sind delikte die der staatsanwalt von a"mts wegen verfolgen mu"B [...] in bei"de fällen hat der staatsanwalt gsacht er verneint des öfentliche interesse

12 Der Beispielfall wird ausführlich erläutert in Spranz-Fogasy (1997), Kap. II.3. 
- bei der Projektion erfolgloser Schlichtungsverhandlung:

C: [...] ăh isch muß dann also dem herrn beck die beschtătigung geben * daß der heutige sủhne*versuch erfolglos verlaufen i"st * âh unt aufgrund dieser bescheinigung ha"t er dann des rescht * beim amtsgerischt * privatklage * gegen sie * zu erheben $[\ldots]$

- bei der Abwehr überzogener Forderungen:

C: [..] schadensersatz [..] der setzt voraus * daß eine unerlaubte handlung im sinne vom acht/ paragraph achthundertdrei"ezwanzisch be ge be * vor*liegt

- beim Diktat des Vergleichsvorschlags:

C: ziffer eins * die antragsgegner des sin also sie * răumen * bei * der verhandlung ein *3,5* daß sie * bei dem vorfall * vom dreiunzwanzigsten viertn $[\ldots]$ sehr erregt * waren *4* unt $* 5^{*} a: h * *$ eine überreaktion eingetretn ist * sie * bedauern die"s $* 7 *$ ziffer zwo $[\ldots]$

In allen Fällen solcher formal-juristischer Kompetenzdemonstration sind regelmäßig Wechsel zum dialektal-umgangssprachlichen bzw. (soweit ihm möglich) hochsprachlichen zu beobachten. Es wird jedoch durch Inhalt und Plazierung der Selbstdarstellungsmittel in seiner Rolle als Schlichter deutlich, daß er damit aufkeimende Konflikte zu ersticken sucht: Mit dem Wechsel zur Hochsprache zieht sich der Schlichter auf seine Kompetenz als Situationsmächtiger zurück und demonstriert seine qua Institution verliehene Machtposition als 'Vertreter des Gesetzes'. Dagegen signalisiert der Wechsel in die dialektale Variante einen Rollenwechsel weg vom Vertreter einer Institution hin zum Alltagsmenschen, der im gleichen Milieu lebt. Entsprechend sind auffällige alltagsweltlich-umgangssprachliche Charakteristika an weniger explosiven Positionen zu finden, an denen der Schlichter sich verständnisvoll zeigen oder auch für Gemeinsamkeit werben kann, so z.B.

- bei Gefahr von übermäßigen Expansionen:

C: $\operatorname{mir}(=$ wir) wolln net bei adam un eva anfangen

- bei der Definition des Konfliktgeschehens:

C: [..] wir sind alle nischt mehr * zwonzich über des alter sin mer alle schon draus * äh wir wissn doch * wo was: * hergsacht wird wird was hingsacht sacht man in pfarrheim * ä:h u:n wahrscheinlich hat jeder so e biBl an an dem ding gedreht * bis es so rischtisch eskaliert i"st [...]

- bei der Aushandlung von Vergleichskomponenten:

C: uff die fuffzeh mark kommt=s a nimmer druff oa 
- bei der Verhandlung über den Vergleichsvorschlag

C: [...] fra neumeier * ein a"lter grundsatz der vergleichsbehörde oder beim * jurist überhaupt ohne jus ke:n schuß uff gut pfarrheimerisch gsacht oh"ne geld ke: musik [...] wie wär $=\mathrm{S}$ * [FRAGEND:] damit a"lles vom disch

Auffällig ist hier, neben der dialektalen Sprachvariante, die häufige Verwendung von Gemeinplätzen, Phrasen und formelhaften Wendungen, die wohl in ihrer dialektalen Variante den Schlichtungsteilnehmern vertraut vorkommen. Die Präsentation von 'Volksweisheiten' als Formen gemeinsamer Wissensbestände bietet den Gesprächsteilnehmern die Möglichkeit von Konsens und Gemeinsamkeit und dient, neben der Fraternisierung des Sprechers mit den anderen, der Akzeptanz von für die Verhandlung nötigen Einsichten.

Kastners Schlichtungshandeln in diesem Gespräch zeichnet sich durch zwei komplementäre Handlungsweisen aus: Kastner kontrolliert das konfliktäre Potential des Interaktionsgeschehens durch gesprächs- und handlungsorganisatorische Steuerungsaktivitäten, und er forciert das Einigungsgeschehen durch geschicktes Aufgreifen, Fokussieren, Ausblenden von Elementen des Konfliktsachverhalts und der Forderungs- bzw. Bereitschaftserklärungen, die er zwischen den Konfliktparteien verschiebt. Dabei nutzt er die selbstdarstellerischen Mittel zur Demonstration formal-juristischer und lebensweltlicher Kompetenz und den Wechsel zwischen beiden Präsentationsformen bzw. zwischen der jeweiligen verbalen Repräsentation durch die Hochsprache und den Dialekt. Die Konfliktparteien lassen sich auf dieses Handeln ein und fördern es, indem sie die Steuerungsaktivitäten akzeptieren und sich auch bereit zeigen, den sukzessive erfolgenden Sachverhaltsdarstellungen einerseits und der daraus entstehenden allmählichen Konstruktion einer Schlichtungsformel andererseits zu folgen.

\section{Aspekte der Selbstdarstellung, Reaktionen und Auswirkungen}

An unseren Beispielen läßt sich zeigen, daß im Hinblick auf die Selbstdarstellung mehrere Faktoren eine Rolle spielen, die wir im folgenden erläutern:

die Interaktionssituation, Anzahl und Gruppierungen der Interaktanten (Aushandeln und Akzeptanz situativer Rahmenvorgaben (6.1)).

die Interaktionsaufgaben und die wechselseitige Akzeptanz rahmengebundener Rollenasymmetrien (gemeinsam Interaktionsaufgaben bearbeiten und Interaktionsziele erreichen (6.2)),

- die wechselseitigen Partnerhypothesen der Sprecherlnnen und deren Vorstellungen darüber beim anderen (negative Partnerhypothesen: nur auf faire Weise Ärger zeigen (6.3)),

- die Kooperativität der TeilnehmerInnen (Kooperativität - Alle tragen zu positiven Selbstdarstellungen bei (6.4)). 


\subsection{Aushandeln und Akzeptanz situativer Rahmenvorgaben}

Sprecher und Sprecherinnen haben vor vielen Gesprächen eine bestimmte Vorstellung von den auf sie zukommenden Gesprächssituationen und ihren Rahmenbedingungen, von ihrer Rolle, die sie innerhalb dieser Interaktion ausfüllen (wollen), davon, welche Aufgaben sie zu erfüllen haben oder welche Position sie vertreten wollen, was die anderen Gesprächsteilnehmer möglicherweise von ihnen erwarten, wie sie ihnen erscheinen mögen usw. Gerade institutionelle oder öffentliche $\mathrm{Ge}$ spräche werden häufig von den Gesprächsteilnehmerlnnen vorbereitet. In der aktuellen Gesprächssituation müssen viele Vorstellungen den Gegebenheiten der Situation angepaßt werden: Die Gesprächsbeteiligten werden, je nach ihrer Rolle und ihren Möglichkeiten, (neue) Situationsdefinitionen, Aufgaben und Ansprüche aushandeln und bearbeiten. Bei diesen Aktivitäten zeigen sich meist schon recht früh die individuellen Argumentations- oder Redestile der Beteiligten, man merkt, mit wem man 'kann' und mit wem nicht, wer eher 'gegen' und wer 'für' einen ist. Die ersten Äußerungen dienen einer wechselseitigen Kategorisierung und Typisierung, und sie dienen der Gruppen- oder Fraktionsbildung.

Dies zeigt sich auch an unseren Beispielen. Im ersten Beispiel der umweltpolitischen Auseinandersetzung vermitteln die Gesprächsbeteiligten über die Inhaite und die präsentierten Gesprächsstile auch so etwas wie 'Parteizugehörigkeit': Sprecher A als jemand, der sich als 'Realist' darstellt mit legitimen Eigeninteressen - kontrastiv zu seiner 'idealistischen' Gegnerin, die Verhaltensveränderungen zugunsten des Umweltschutzes erste Priorität einräumt. Ganz anders ist die Darstellung eines positiven Selbst im zweiten Beispiel, in dem der Situationsmächtige bereits zu Beginn der Schlichtung durch seine häufigen Kodewechsel ein bestimmtes, eher kooperatives Interaktionsklima herstellt.

\subsection{Gemeinsam Interaktionsaufgaben bearbeiten und Interaktionsziele erreichen}

Viele Kommunikationssituationen weisen Rollenasymmetrien auf. Die Inhaber einer im Vergleich zu anderen Gesprächsbeteiligten bevorzugten Gesprächsrolle (sei es qua Institution situationsmächtig wie ein Schlichter, Lehrer oder Prüfer oder aufgrund anderer Rahmenvorgaben wie bei Moderatoren, Beratern oder Therapeuten) sollten sich um ein sinnvolles 'Ausnutzen' dieser Asymmetrie bemühen. Signalisieren Situationsmächtige die Bereitschaft zu Kooperation und Perspektivenübernahme und nutzen sie ihre bevorzugte Position nur im Rahmen des für die anderen Gesprächsteilnehmerlnnen Akzeptablen aus, so dient dies auch eigener positiver Selbstdarstellung. Dies gilt auch für kritische Situationen: In einem anderen Schlichtungsgespräch demonstrieren Schlichter und Beklagte Kooperativität und Bereitschaft zur Perspektivenübernahme, wohingegen die Klägerin aufgrund einer anderen Handlungsorientierung sowohl den Schlichter als auch die Beklagte wiederholt angreift und beschuldigt. Dies hat zur Folge, daß sie als Klägerin und als Gesprächspartnerin nach und nach nicht mehr glaubwürdig bzw. akzeptabel ist. Dagegen vermitteln demonstrierte Geduld des Schlichters und die Zurückhaltung der Beklagten positive Selbstdarstellungen.

Eine untergeordnete Gesprächsrolle - wie beim Prüfling, bei Klägern und Beklagten im Schlichtungsverfahren oder auch beim einzelnen Vertreter einer Position gegenüber einer ganzen Reihe von Kontrahenten etc. - zeichnet sich meist dadurch 
aus, daß die untergeordnet Beteiligten im Hinblick auf die Gesprächssituation nicht die entsprechende Routine mitbringen, in irgendeiner Form 'etwas zu verlieren haben' oder sich 'stärkeren' InteraktionspartnerInnen gegenüber sehen. Da bedarf es eines höheren Aufwands für eine positive Selbstdarstellung, denn es geht in den benachteiligten Rollen häufig um die Bearbeitung von zwei Aufgaben. Zum einen um die in der Interaktionssituation aktuell zu bearbeitende Aufgabe: Eine Prüfungsfrage soll beantwortet werden, ein Sachverhalt soll nachvollziehbar dargestellt, einem Argument soll begegnet werden. Zum zweiten soll mit dem Lösen der Prüfungsaufgabe auch Kompetenz oder Sicherheit gezeigt, mit der Darstellung eines Sachverhalts auch Glaubwürdigkeit vermittelt werden, und bei einer Position in der Minderheit darf man sich mögliche Nervosität in der Rolle des Schwächeren nicht anmerken lassen.

\subsection{Negative Partnerhypothesen: Nur auf faire Weise Ärger zeigen}

Die Manifestation negativer Emotionen, wie Ärger, der auf das Gegenüber gerichtet ist, wirkt sich häufig dann negativ auf die Selbstdarstellung aus, wenn sie mit unfairen Strategien verwoben ist, z.B. der strategischen Demonstration von Expertentum zur Demonstration von Überlegenheit. Umgekehrt formuliert: Die Darstellung einer bestimmten Position, Argumentation oder Einstellung kann die positive Präsentation des Selbst ins Negative verkehren, wenn erkennbar weitere Aufgaben zu Lasten des Gegenübers auf der Beziehungsebene bearbeitet werden, beispielsweise durch die Demonstration von Überlegenheit zur Imagereparatur oder von Ärger, Angriffen und Schuldzuweisungen im Kontext von Problembearbeitungen.

\subsection{Kooperativität - Alle tragen zu positiven Selbstdarstellungen bei}

Daß eine gelungene Selbstdarstellung nicht nur von den eigenen Aktivitäten abhängt, sondern auch von den Aktivitäten der anderen Gesprächsteilnehmerlnnen, zeigt das zweite Beispiel: Die Konfliktparteien verhalten sich dem Schlichter gegenüber kooperativ - und reagieren in gewisser Weise auf die kooperativen Aktivitäten des Schlichters. Dadurch, daß dieser wiederholt die dialektale Variante in Verbindung mit verständnissignalisierenden Aktivitäten verwendet, demonstriert er auch seine Bereitschaft zur Perspektivenübernahme, die die anderen Gesprächsbeteiligten dadurch honorieren, daß sie seine Situationsdominanz als Schlichter akzeptieren.

Keinem gelingt es, ein positives Selbstbild bzw. eine positive Selbstdarstellung zu präsentieren, wenn es die anderen nicht zulassen. ${ }^{13}$ Unsere Beispiele zeigen, wie sehr die jeweilige Selbstdarstellung auch mit der Kooperativität - oder auch mit deren Mangel - der anderen Gesprächsteilnehmerlnnen zusammenhängt: Tragen diese durch entsprechende positive Reaktionen die Selbstdarstellung des Sprechers oder der Sprecherin mit, so fördern sie damit auch eine positive wechselseitige Selbst- und Fremddarstellung. Greifen die anderen Gesprächsteilnehmerlnnen einen

13 Hier sind immer auch Unterschiede bei dyadischen bzw. Mehrpersonengesprächen zu erwarten: vgl. dazu Petter-Zimmer (1990). 
Sprecher oder eine Sprecherin dagegen in irgendeiner Form an oder melden Zweifel an der Glaubwürdigkeit oder Integrität ihres Gegenübers an, so bedarf es auf seiten des Ausgegrenzten eines besonderen Aufwandes, Fingerspitzengefühls und Besonnenheit, um seine Selbstdarstellung noch zu retten.

Gesprächspartnerlnnen reagieren auf und operieren mit den (bewußten oder unbewußten) Fremd- und Selbstdarstellungen der anderen. Sie akzeptieren, stützen, problematisieren, unterlaufen oder bekämpfen sie. Zugleich werden damit Kompetenz und Glaubwürdigkeit gewürdigt, angezweifelt oder abgesprochen. Entsprechend solchen Reaktionen müssen die Gesprächsbeteiligten ihre Selbstdarstellung anpassen, wirksame Mittel verstärken oder ggf. strategische Wechsel in ihrer Präsentation vornehmen. Dabei ist zu berücksichtigen, daß gerade Selbstdarstellungsformen einer starken, selbststabilisierenden Eigendynamik unterworfen sind, da Gesprächsteilnehmer gegenüber anderen wichtigen Gesprächselementen (wie 2.B. handlungsorganisatorische oder inhaltliche Entwicklungen) als stabile Faktoren behande/t werden. Die Stabilität der physischen Erscheinung wird weitgehend auch auf Eigenschaften von Teilnehmern übertragen. Dies hat zur Folge, daß Veränderungen des Selbstbildes nur in geringem Maße und nur in eher randständigen Bereichen möglich ist.

Gerade Reaktionen auf die Selbstdarstellung anderer, die eher kritischen Charakter haben, bewirken vielfach Verschiebungen des Interaktionsfokus beispielsweise zur metakommunikativen Behandlung des auftauchenden Interaktionsproblems oder durch einen Modalitätenwechsel (2.B. zu Ironie oder zu direkten Angriffen). Metakommunikative Behandlungen von Selbstdarstellung haben jedoch. wie alle metakommunikativen Aktivitäten, vielfach die Qualität einer Ultima Ratio und produzieren von daher in besonderer Weise ein Gefährdungspotential für die Interaktion selbst. ${ }^{14}$ Ähnlich sind auch Modalitätenwechsel problematisch und kritisch für die Interaktion, weil deren Implikationen und Präsuppositionen oft undurchsichtig sind und dementsprechend die Beteiligten mit 'falschen Voraussetzungen' weiter interagieren. Dadurch kann es in den Gesprächen oder in der Behandlung von handlungsschematischen Aufgaben zu Expansionen, Stagnationen oder zu Rückschritten kommen bis hin zum Gesprächsabbruch. Umgekehrt fördern positive, also akzeptierende oder gar stützende Reaktionen auf die Selbstdarstellung eines Interaktionsteilnehmers die Gesprächsdynamik und das Gesprächsklima.

\section{Ein bewußterer Umgang mit Selbstdarstellung}

Ein bewußterer Umgang mit Selbstdarstellung in Gesprächen ist kaum als gezielt strategische Wahl von Selbstdarstellungsmitteln möglich. Solchermaßen bloß 'aufgesetzte' Eigenschaften oder Merkmale lassen sich in der Dynamik einer Interaktion selten durchhalten, und die daraus sich ergebenden Brechungen des Interaktionshandelns sind dann um so (interaktions-)kritischer. Es ist daher wichtig, die sozialen Situationen, in denen man sich befindet, richtig 'lesen' zu können und die eigenen Möglichkeiten angemessen einzupassen. Dazu gehört, sich der anstehenden Interaktionsaufgaben zu vergewissern, sich über die eigene Beteiligungsrolle und die der

\footnotetext{
14 Vgl. dazu Deppermann (1997); dort auch ausführliche Analysen zur Rhetorik der Glaubwürdigkeit, zu elementaren Sequenzen und gesprächsdynamischen Folgen der Glaubwürdigkeitsbearbeitung.
} 
anderen wie auch über die jeweiligen sozialen und individuellen Qualitäten bewußt zu sein bzw. zu werden und den jeweiligen Stand und die Entwicklung des Gesprächs aufmerksam zu beobachten. Kooperative Aktivitäten wie die Demonstration von Perspektivenübernahme oder der Bereitschaft dazu, die Würdigung des Gesprächspartners als akzeptablen Gesprächspartner oder das Einhalten der Höflichkeitsregeln wirken sich dann positiv im Sinne der Selbstdarstellung aus (allerdings nicht im Verein mit strategisch verdeckten partnerverletzenden oder unfairen Aktivitäten).

Die Selbstdarstellung in Gesprächen ist immer einer Reihe von Unwägbarkeiten ausgesetzt, wir können nicht immer wissen und kontrollieren, was wir tun, wir können Reaktionen anderer nicht programmieren, und wir sind den strukturellen Voraussetzungen und Gefahren der Situationstypik und des Interaktionsprozesses ausgesetzt. So bergen beinahe alle Selbstdarstellungsmittel auch paradoxe, funktionale wie dysfunktionale Potentiale in sich:

- Entgegenkommen in Verhandlungen kann auch als Schwäche interpretiert werden;

- die Demonstration von Durchsetzungwillen kann als Sturheit ${ }^{15}$ aufgefaßt werden;

- Submissivität eines Bewerbers in Bewerbungsgesprächen kann als mangelnde Initiative gelten:

- Initiative kann als Dominanz ausgelegt werden;

- Souveränität eines Prüflings kann übersteigerte Erwartungen wecken; Zurückhaltung dagegen kann umgekehrt Hilfestellungen erzeugen;

Die Demonstration von Expertenschaft durch Präsentation von Fachwissen oder durch Verwendung von Fachterminologie ${ }^{10}$ kann Unverständlichkeit und Reaktanz hervorrufen oder in Langeweile und Ignoranz münden usw.

Oder, um das obige Beispiel des Beraters/Therapeuten funktional umzukehren:

- Häufige Unterbrechungen können auch als Zeichen von Interesse gelten, und

- direktives Fragen kann als Ausdruck fachlicher Kompetenz interpretiert werden.

Die interaktive Selbstdarstellungsarbeit zeigt sich so als ständiger und schwieriger Balanceakt für Gesprächsteilnehmerlnnen, bei dem es wichtig ist, die Reaktionen anderer zu antizipieren und auf die Reaktionen der GesprächspartnerInnen flexibel zu reagieren. Selbstdarstellung in Gesprächen erfordert ständige Balance und Anpassung des eigenen Handelns und dies - besonders in öffentlichen und beruflichen Gesprächen - unter dem Gesichtspunkt der besonderen Interaktionsaufgaben, der

\footnotetext{
15 Vgl. Pruitt/Smith 1981.

${ }_{18} \mathrm{Vgl.} \mathrm{dazu} \mathrm{die} \mathrm{Charakterisierung} \mathrm{der} \mathrm{"Expertenrede"} \mathrm{in} \mathrm{Spiegel} \mathrm{(1997).}$
} 
individuellen Ziele und der eigenen wie der gemeinsamen Erfolgsorientierung. Unser Beitrag kann dafür zwar keine Patentrezepte bieten, dagegen sprechen starke Situationsabhängigkeit und die Besonderheiten des jeweils individuellen Teilnehmers. Er zeigt jedoch, um die Wahrnehmung dafür zu sensibilisieren, einige wichtige Aspekte und Dimensionen der Selbstdarstellung auf und demonstriert beispielhaft, in welcher Weise Gesprächsaktivitäten von - positiver wie negativer - Bedeutung für die Selbstdarstellung und damit für den Erfolg und Mißerfolg interaktiven Handelns sein können.

\section{Transkriptionszeichen}

GROSSBUCHSTABEN

\# \#

K

[...]

(...)

Unterstreichung

Unterstreichung

(schon mal)

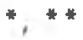

* 2,5 *

$=$

$$
\text { I }
$$

Kommentare

Extension eines Kommentars

(zugehörige) Kommentarzeile

Auslassung des Autors

unverständliche Passagen

Simultanpassagen

fragwürdiges Wortverständnis

Mikropause (unter 1 Sekunde)

Pause mit Sekundenangabe

schwebende Intonation

Betonung

Dehnung

Verschleifung

Wortabbruch

\section{Literatur}

Balint, M. (1965). Der Arzt, sein Patient und die Krankheit. Stuttgart.

Deppermann, A. (1997). Glaubwürdigkeit im Konflikt. Rhetorische Techniken im Streit. Frankfurt a.M.

Deppermann, A. \& Spranz-Fogasy, T. (i.Dr.). Kommunikationsstörungen durch den Gesprächsprozeß. In: Fiehler, R. (Hrsg.), Verständigungsprobleme und gestörte Kommunikation. Opladen. 
Fiehler, R. (1990). Kommunikation und Emotion. Berlin.

Gumperz, J. (1982). Discourse strategies. Cambridge.

Henne, H. \& Rehbock, H. (1982). Einführung in die Gesprächsanalyse. Berlin

Hinnenkamp, V. \& Selting, M. (Hrsg.)(1989). Stil und Stilisierung. Arbeiten zur interpretativen Soziolinguistik. Tübingen.

Holly, W. (1979). Imagearbeit in Gesprächen. Zur linguistischen Beschreibung des Beziehungsaspekts. Tübingen.

Holly, W. (1990). Politikersprache. Inszenierungen und Rollenkonflikte im informellen Sprachhandeln eines Bundestagsabgeordneten. Berlin.

Hundsnurscher, F. (1994). Dialog-Typologie. In: Fritz, G. \& Hundsnurscher, F. (Hrsg.), Handbuch der Dialogforschung. Tübingen, 203-238.

Huttner, G. (1981). Das Sühneverfahren in Baden-Württemberg. Stuttgart (Schriftenreihe der Funkstelle).

Kallmeyer, W. (1988). Konversationsanalytische Beschreibung. In: Ammon, U., Dittmar, N. \& Mattheier, K. (Hrsg.), Handbuch Soziolinguistik. Ein internationales Handbuch zur Wissenschaft von Sprache und Gesel/schaft. Berlin/New York, Artikel 121.

Kallmeyer, W. (1995). Zur Darstellung von Kommunikativem sozialen Stil in soziolinguistischen Gruppenporträts. In: Keim, I., Kommunikative Stilistik einer sozialen Welt "kleiner Leute ". Kommunikation in der Stadt. Bd. 4.3. Hrsg. v. Kallmeyer, W. Berlin.

Kallmeyer, W. \& Schütze, F. (1976). Konversationsanalyse. In: Studium Linguistik 1, 1-28.

Keim, I. (1995). Kommunikative Stilistik einer sozialen Welt "kleiner Leute". Kommunikation in der Stadt. Bd. 4.3. Hrsg. v. Kallmeyer, W. Berlin.

Munsberg, K. (1994). Mündliche Fachkommunikation: Das Beispiel Chemie. Tübingen.

Nothdurft, W. \& Spranz-Fogasy, T. (1991). Gesprāchsanalyse von Schlichtungs-Interaktion. Methodische Probleme und ihre Hintergründe. In: Flader, D. (Hrsg.), Verbale Interaktion: Studien zur Empirie und Methodologie der Pragmatik. Stuttgart, 222-240.

Petter-Zimmer, Y. (1990). Politische Fernsehdiskussionen und ihre Adressaten. Tübingen.

Pruitt, D.G. \& Smith, D.L. (1981). Impression management in bargaining: Images of firmness and trustworthiness. In: Tedeschi, J.T. (Ed.l, Impression managment theory and social psychology research. New York, 247-267.

Rogers, C. R. (1988). Die klientenzentrierte Gesprächspsychotherapie. Frankfurt a.M.

Sandig, B. (1986). Stilistik der deutschen Sprache. Berlin.

Sandig, B. (1995). Tendenzen der linguistischen Stilforschung. In: Stickel, G. (Hrsg.), Stilfragen Berlin, 27-61.

Schmitt, R. (1992). Die Schwellensteher. Sprachliche Präsenz und sozialer Austausch in einem Kiosk. Tübingen.

Schwitalla, J. (1996). Beziehungsdynamik. Kategorien für die Beschreibung der Beziehungsgestaltung sowie der Selbst- und Fremddarstellung in einem Streit- und Schlichtungsgespräch. In: Kallmeyer, W. (Hrsg.), Gesprächsrhetorik. Rhetorische Verfahren im Gesprächsprozess. Tübingen, 279-349.

Spiegel, C. (1995). Streit. Eine linguistische Untersuchung verbaler Interaktionen in alltäglichen Zusammenhängen. Tübingen.

Spiegel, C. (1997). Selbst- und Fremdstilisierungen in umweltpolitischen Auseinandersetzungen. In: Sandig, B. \& Selting, M. (Hrsg.), Sprech-und Gesprächsstile. Berlin, 286-317.

Spranz-Fogasy, T. (1988). Medikamente im Gespräch zwischen Arzt und Patient - Gesprächsanalysen für die Praxis. In: Deutsche Sprache 3, 240-258.

Spranz-Fogasy, T. (1992). Ärztliche Gesprächsführung - Inhalte und Erfahrungen gesprächsanalytisch fundierter Weiterbildung. In: Fiehler, R. \& Sucharowski, W. (Hrsg.), Kommunikationsberatung und Kommunikationstraining. Opladen, 68-78.

Spranz-Fogasy, Th. (i.Dr.). Interaktionsprofile. Die Herausbildung individueller Handlungstypik in Gesprächen. Habilitationsschrift. Opladen. 